\title{
TOPOLOGIES OF POINTWISE CONVERGENCE ON FAMILIES OF EXTREMAL POINTS AND WEAK COMPACTNESS
}

\author{
IAN TWEDDLE
}

\begin{abstract}
Bourgain and Talagrand showed that a bounded subset of a Banach space is weakly relatively compact provided it is relatively countably compact for the topology of pointwise convergence on the extremal points of the closed unit ball in the dual space. We give a version of this result for quasi-complete locally convex spaces. We also consider situations where the completeness or boundedness assumptions may be relaxed.
\end{abstract}

Let $E$ be a Hausdorff locally convex space with dual $E^{\prime}$ and let $S$ be a family of subsets of $E^{\prime}$ such that

(i) each $A \in S$ is $\sigma\left(E^{\prime}, E\right)$-compact and absolutely convex,

(ii) $\bigcup\{A: A \in S\}$ spans $E^{\prime}$.

Let $F^{\prime}$ be the vector subspace of $E^{\prime}$ spanned by the extremal points of the elements of $S$ (cf. $[6, \S 2])$.

THEOREM. Suppose that $E$ is quasi-complete under its Mackey topology $\tau\left(E, E^{\prime}\right)$. If $X$ is a bounded subset of $E$ which is relatively countably compact under $\sigma\left(E, F^{\prime}\right)$, then $X$ is relatively compact under $\sigma\left(E, E^{\prime}\right)$.

ProOF. Let $\left\{x_{n}\right\}$ be any sequence in $X$ and let $x$ be a $\sigma\left(E, F^{\prime}\right)$-cluster point of $\left\{x_{n}\right\}$. By Eberlein's theorem $[3, \S 24.2(1)]$ it is enough to show that $x$ is also a $\sigma\left(E, E^{\prime}\right)$-cluster point of $\left\{x_{n}\right\}$. Suppose that this is false. Then we can find a finite subset $\left\{x_{1}^{\prime}, x_{2}^{\prime}, \ldots, x_{m}^{\prime}\right\}$ of $E^{\prime}$ such that $x_{n} \notin x+\left\{x_{1}^{\prime}, x_{2}^{\prime}, \ldots, x_{m}^{\prime}\right\}^{\circ}$ except for finitely many $n(*)$. Also we can choose $A_{1}, A_{2}, \ldots, A_{p}$ say in $S$ such that each $x_{r}^{\prime}$ is in the span of $A_{1} \cup A_{2} \cup \cdots \cup A_{p}$. If $B=A_{1}+A_{2}+\cdots+A_{p}$ then $B$ is an absolutely convex $\sigma\left(E^{\prime}, E\right)$-compact set whose extremal points are of the form $a_{1}^{\prime}+a_{2}^{\prime}+\cdots+a_{p}^{\prime}$, where $a_{r}^{\prime}$ is an extremal point of $A_{r}(r=1,2, \ldots, p)$. Let $G^{\prime}$ be the linear span of $B$, let $q: E \rightarrow E / G^{\prime \circ}$ be the quotient map and let $G$ be the Banach space obtained by completing $E / G^{\prime \circ}$ with respect to the norm defined by taking $B$ as the closed unit ball of $G^{\prime}$. We can apply Théorème 1 of $[1]$ to deduce that $q(X)$ is $\sigma\left(G, G^{\prime}\right)$-relatively compact. Since $\sigma\left(G, G^{\prime}\right)$ and the topology of pointwise convergence on the extremal points of $B$ must then coincide on $q(X)$, it follows that $q(x)$ is a $\sigma\left(G, G^{\prime}\right)$-cluster point of $\left\{q\left(x_{n}\right)\right\}$. This contradicts (*).

If we do not assume quasi-completeness in the theorem, the argument still shows that $X$ is relatively countably compact under $\sigma\left(E, E^{\prime}\right)$. In some instances this is sufficient for weak relative compactness. Thus we have, in particular, by $[\mathbf{4}$, Theorem 4.2]

Received by the editors August 12, 1981 and, in revised form, November 18, 1981.

1980 Mathematics Subject Classification. Primary 46A50; Secondary 46A06, 46A20, 46A55. 
COROLlARY 1. Suppose that $E$ is metrizable. If $X$ is a bounded subset of $E$ which is (relatively) countably compact under $\sigma\left(E, F^{\prime}\right)$, then $X$ is (relatively) compact under $\sigma\left(E, E^{\prime}\right)$.

If $Y$ is relatively countably compact under $\sigma\left(E, F^{\prime}\right)$ and convex then $Y$ is bounded in the strong topology $\beta\left(E, F^{\prime}\right)$. (We cannot find a precise reference for this although we believe that it is standard. It is enough to prove the assertion when $0 \in Y$ and Proof (b) of the Banach-Mackey theorem in [3, §20.11(3)] may be adapted for this case.) This implies that $Y$ is $\sigma\left(E, E^{\prime}\right)$-bounded and therefore bounded for the original topology of $E$. We then have the following variant of [5, Theorem 2]:

COROLLARY 2. The same convex subsets of $E$ are (relatively) countably compact for the topologies $\sigma\left(E, F^{\prime}\right)$ and $\sigma\left(E, E^{\prime}\right)$.

REMARKS. (i) Corollary 1 shows that Théorème 1 of [1] is valid for an arbitrary normed space.

(ii) Let $H^{\prime}$ be the dual of $E$ under the topology of uniform convergence on the $\sigma\left(E^{\prime}, E\right)$-compact sets. Then $x^{\prime} \in H^{\prime}$ if and only if $x^{\prime}$ is an element of the $\sigma\left(E^{*}, E\right)$ closed absolutely convex envelope $A$ of some $\sigma\left(E^{\prime}, E\right)$-compact set $C$. Such a set $A$ is $\sigma\left(H^{\prime}, E\right)$-compact and its extremal points are elements of $E^{\prime}$ [3, §25.1(7), (8)]. Thus $\sigma\left(E, E^{\prime}\right)$ corresponds to $\sigma\left(E, H^{\prime}\right)$ in the same way as $\sigma\left(E, F^{\prime}\right)$ corresponds to $\sigma\left(E, E^{\prime}\right)$ and we may for example add $\sigma\left(E, H^{\prime}\right)$ to the list of topologies in Corollary 2.

(iii) Recently in [2] Khurana has given a new proof of Théorème 1 of [1] using the Choquet integral representation theorem.

\section{REFERENCES}

1. J. Bourgain and M. Talagrand, Compacité extrêmale, Proc. Amer. Math. Soc. 80 (1980), 68-70.

2. S. S. Khurana, Pointwise compactness on extreme points, Proc. Amer. Math. Soc. 83 (1981), 347-348.

3. G. Köthe, Topological vector spaces. I, Die Grundlehren der mathematischen Wissenschaften 159, Springer-Verlag, Berlin-Heidelberg-New York, 1969.

4. J. D. Pryce, $A$ device of $R$. J. Whitley's applied to pointwise compactness in spaces of continuous functions, Proc. London Math. Soc. (3) 23 (1971), 532-546.

5. I. Tweddle, Weak compactness in locally convex spaces, Glasgow Math. J. 9 (1968), 123-127.

6. —_, Vector-valued measures, Proc. London Math. Soc. (3) 20 (1970), 469-489.

Department of Mathematics, University of StiRling, Stirling FK9 4LA, SCOTLAND, U.K. 